\title{
SPECTRAL COLLOCATION METHODS FOR A PARTIAL INTEGRO-DIFFERENTIAL EQUATION WITH A WEAKLY SINGULAR KERNEL
}

\author{
CHANG HO KIM' ${ }^{1}$ and $\mathrm{U}$ JIN CHOI ${ }^{2}$
}

(Received 7 December 1993; revised 28 February 1997)

\begin{abstract}
We propose and analyze the spectral collocation approximation for the partial integrodifferential equations with a weakly singular kernel. The space discretization is based on the pseudo-spectral method, which is a collocation method at the Gauss-Lobatto quadrature points. We prove unconditional stability and obtain the optimal error bounds which depend on the time step, the degree of polynomial and the Sobolev regularity of the solution.
\end{abstract}

\section{Introduction}

Let $\Omega$ be a rectangular domain in $\mathbb{R}^{2}$ with boundary $\partial \Omega$ (typically $\Omega \equiv(-1,1)^{2}$ ), and let $T \in \mathbb{R}$ satisfy $0<T<\infty$. We shall consider spectral methods for the following integro-differential equation with a weakly singular kernel:

$$
\begin{aligned}
u_{t}-\Delta u & =\int_{0}^{t} K(t-s) B(t, s) u(s) d s+f(\mathbf{x}, t), \quad(\mathbf{x}, t) \in \Omega \times(0, T], \\
u & =0 \quad \text { on } \partial \Omega, \quad t>0 \\
u(\cdot, 0) & =u_{0} \quad \text { in } \quad \Omega
\end{aligned}
$$

where $B(t, s)$ is a general partial differential operator of second order with smooth coefficients

$$
B(t, S)=-\sum_{i . j=1}^{2} \frac{\partial}{\partial x_{j}}\left(b_{i j}(\mathbf{x}, t, s) \frac{\partial}{\partial x_{i}}\right)+\sum_{j=1}^{2} b_{i j}(\mathbf{x}, t, s) \frac{\partial}{\partial x_{j}}+b_{0}(\mathbf{x}, t, s) I(
$$

'Current address: Dept. of Applied Mathematics, Konkuk University, Chungju, Chungbuk 380-701, Korea.

${ }^{2}$ Department of Mathematics of KAIST, Kusong-Dong 373-1, Yousong-Gu, Taejon 305-701, Korea.

(C) Australian Mathematical Society, 1998, Serial-fee code 0334-2700/98 
and $K$ is a weakly singular kernel such that

$$
|K(t)| \leq C t^{-\mu} \quad \text { with } \quad 0 \leq \mu<1, \quad \text { for } t>0 .
$$

Further, throughout this paper, we shall assume that $f$ is sufficiently smooth.

Partial integro-differential equations of this nature appear in applications such as heat conduction in materials with memory, population dynamics, viscoelasticity and theory of nuclear reactors (see the introduction in Yanik and Fairweather [13], where references to studies of existence, uniqueness and regularity are also given).

For a numerical solution of (1.1), both the finite element and finite difference methods have been considered by several authors. Thomée and Zhang [12], Chen et al. [5] and Pani et al. $[9,10]$ have derived optimal error estimates both for semidiscrete and fully discrete finite elements and finite difference methods. In this paper, we propose and analyze the spectral collocation approximation for the partial integrodifferential equations with a weakly singular kernel.

Spectral methods are classical and widely used techniques to solve differential equations, both theoretically and numerically. These methods appear to be competitive with both the finite difference methods and the finite element methods. They must be decisively preferred to the latter whenever the solution is highly regular and the geometric dimension of the domain becomes large. Moreover, by these methods, it is possible to control easily the solution of those numerical problems affected by oscillation and instability phenomena. The use of spectral and pseudo-spectral methods in computations for many fields of engineering has been matched by deeper theoretical studies $[3,6]$. Recently, spectral methods have been studied by variational techniques, to point out the dependence of the approximation error (for instance in the $L^{2}$-norm, or in the energy norm) on the regularity of the solution and the discretization parameter. Indeed, often the solution is not infinitely differentiable (see Canuto and Quarteroni [4]). Spectral methods involve expansions in the basis of polynomials (or trigonometric polynomials) that are orthogonal with respect to a weighted measure. The popularly used basis is either the family of Legendre polynomials or the family of Chebyshev polynomials. In practice, spectral methods are implemented via collocation techniques, which are discretizing methods involving Gauss-type quadrature nodes. Particularly in the Chebyshev case, the use of the fast Fourier transforms allows a less expensive computation time for the derivatives and the nonlinear terms. The aim of this paper is the numerical analysis of the collocation methods at the Gauss-Lobatto nodes including Legendre and Chebyshev nodes, for some kinds of partial integro-differential equations in the square $(-1,1)^{2}$.

An outline of this paper is as follows. In Section 2, we first introduce the weighted Sobolev spaces on a square associated with the Jacobi weighted measure. We define several projection operators from weighted spaces onto the space of polynomials with degree less than an integer $N$. Section 3 is related to the approximation of (1.1) by the 
Galerkin spectral method and the Ritz-Volterra projection. As has been emphasized in the literature, this method allows one to achieve high accuracy for smooth solutions. In Section 4, we establish stability and convergence of the collocation method for (1.1). Finally in Section 5, the pseudo-spectral solution is advanced in the time direction by using the backward difference method.

\section{Preliminaries}

We introduce some definitions and recall some basic results which will be used throughout the paper. We first introduce the weighted Sobolev spaces on the square associated with the Jacobi weighted measure. For any $\mathbf{x}=\left(x_{1}, x_{2}\right) \in \Omega$, we set $w_{\alpha}(\mathbf{x})=\left(1-x_{1}^{2}\right)^{\alpha}\left(1-x_{2}^{2}\right)^{\alpha}$, where $-1<\alpha<1$. We define

$$
L_{w_{\alpha}}^{2}(\Omega)=\left\{v: \Omega \rightarrow \mathbb{R} \text { measurable; } \int_{\Omega} v^{2}(\mathbf{x}) w_{\alpha}(\mathbf{x}) d \mathbf{x}<+\infty\right\},
$$

which is a Hilbert space for the scalar product

$$
(u, v)_{w_{\alpha}}=\int_{\Omega} u(\mathbf{x}) v(\mathbf{x}) w_{\alpha}(\mathbf{x}) d \mathbf{x} .
$$

For any integer $m \geq 0$, the weighted Sobolev space is defined by

$$
H_{w_{\alpha}}^{m}(\Omega)=\left\{v \in L_{w_{a}}^{2}(\Omega) ; \frac{\partial^{p+q} v}{\partial x_{1}^{p} \partial x_{2}^{q}} \in L_{w_{\alpha}}^{2}(\Omega),(p, q) \in N^{2}, p+q \leq m\right\},
$$

which is equipped with the norm

$$
\|v\|_{m, w_{\alpha} . \Omega}=\left(\int_{\Omega} \sum_{p+q \leq m}\left(\frac{\partial^{p+q} v}{\partial x_{1}^{p} x_{2}^{q}}\right)^{2} w_{\alpha}(\mathbf{x}) d \mathbf{x}\right)^{1 / 2}
$$

and with the semi-norm

$$
|v|_{m, w_{\alpha}, \Omega}=\left(\int_{\Omega} \sum_{p+q=m}\left(\frac{\partial^{p+q} v}{\partial x_{1}^{p} x_{2}^{q}}\right)^{2} w_{\alpha}(\mathbf{x}) d \mathbf{x}\right)^{1 / 2} .
$$

For a real number $s \geq 0$ which is not an integer, the Hilbert space $H_{w_{q}}^{s}(\Omega)$ is defined by interpolation between $H_{w_{\alpha}}^{[s]}(\Omega)$ and $H_{w_{\alpha}}^{[s]+1}$ where $[s]$ is the integer part of $s$, and its norm is denoted by $\|\cdot\|_{s, w_{\alpha}, \Omega}$. We denote by $H_{w_{\alpha}, 0}^{s}(\Omega)$ the closure in $H_{w_{\alpha}}^{s}(\Omega)$ of the space $D(\Omega)$ of all functions of $C^{\infty}$ having a compact support in $\Omega$. Whenever there is no confusion, we drop the subscript $\Omega$ from $\|\cdot\|_{m, w_{\alpha}, \Omega}$ and $(\cdot, \cdot)_{m, w_{\alpha} . \Omega}$. Throughout this paper, we denote by $C$ generic constants independent of $N$. 
We set

$$
A_{w_{\alpha}}(u, v)=\int_{\Omega} \nabla u \cdot \nabla\left(v w_{\alpha}\right) d \mathbf{x} \quad \text { for any } u, v \in H_{w_{\alpha}}^{1}(\Omega) .
$$

A complete study of the properties of the bilinear form $A_{w_{\alpha}}(\cdot, \cdot)$ has been done in [1] for one or two-dimensional problems. We report hereafter some results of this analysis. There exists a positive constant $\beta$ such that

$$
\left|A_{w_{\alpha}}(u, v)\right| \leq \beta\|\nabla u\|_{w_{\alpha}}\|\nabla v\|_{w_{\alpha}} \quad \text { for any } u, v \in H_{w_{\alpha} .0}^{1}(\Omega) .
$$

Moreover, for any $v \in H_{w_{\alpha}, 0}^{1}(\Omega)$, there exists a constant $c>0$ such that

$$
\left|A_{w_{\alpha}}(v, v)\right| \geq c\|\nabla v\|_{w_{\alpha}}^{2}
$$

We also recall the Poincaré inequality

$$
\exists \delta>0:\|v\|_{w_{o}} \leq \delta\|\nabla v\|_{w_{\alpha}} \quad \text { for any } v \in H_{w_{a} .0}^{1}(\Omega),
$$

by which we deduce that $A_{w_{\alpha}}(\cdot, \cdot)$ is coercive, namely

$$
\exists c>0: A_{w_{\alpha}}(v, v) \geq c\|v\|_{1, w_{\alpha}}^{2} \quad \text { for all } v \in H_{w_{\alpha} .0}^{1}(\Omega) .
$$

For an integer $N>0$, we set $\mathbb{P}_{N}=\tilde{\mathbb{P}}_{N} \times \tilde{\mathbb{P}}_{N}$, where $\tilde{\mathbb{P}}_{N}$ is the space of the polynomials of degree $N$ in single variables. Further, we set $\mathbb{P}_{N}^{0}(\Omega)=\left\{p \in \mathbb{P}_{N} \mid p(\mathbf{x})=0\right.$ on $\left.\partial \Omega\right\}$.

For our work, we require some spectral projection operators. First define the orthogonal projection operator $\mathbb{P}_{N}: L_{w_{\alpha}}^{2} \mapsto \mathbb{P}_{N}^{0}(\Omega)$ by

$$
\left(v-P_{N} v, \phi\right)_{w_{a}}=0, \quad \forall \phi \in \mathbb{P}_{N}^{0}(\Omega) .
$$

The projection error is estimated as follows (see [1, 4]):

$$
\left\|v-P_{N} v\right\|_{w_{\alpha}} \leq C N^{-\sigma}\|v\|_{\sigma, w_{\sigma}}, \quad \forall v \in H_{w_{\alpha}}^{\sigma}(\Omega), \quad \sigma>0 .
$$

We define the Ritz projection operator $\Pi_{N}: H_{w_{\alpha} .0}^{1} \mapsto \mathbb{P}_{N}^{0}(\Omega)$ by

$$
A_{w_{a}}\left(v-\Pi_{n} v, \phi\right)=0, \quad \forall \phi \in \mathbb{P}_{n}^{0}(\Omega) .
$$

The error estimate of the Ritz projection can be found in [1,2]: for all $v \in H_{w_{\alpha}}^{\sigma}(\Omega) \cap$ $H_{w_{\alpha}, 0}^{1}(\Omega)$ with $0 \leq \mu \leq \sigma, \sigma \geq 1$

$$
\left\|v-\Pi_{N} v\right\|_{\mu, w_{\alpha}} \leq C N^{e(\mu)-\sigma}\|v\|_{\sigma, w_{\alpha}}
$$

where $e(\mu)=\mu$ if $\mu \leq 1$ and $e(\mu)=2 \mu-1$ if $\mu>1$.

We shall need the following two lemmas from [5]. 
LEMMA 1 (Chen et al. [5]). Assume that y is a nonnegative function in $L_{1}(0, T)$ which satisfies

$$
y(t) \leq b(t)+\beta \int_{0}^{t}(t-s)^{-\alpha} y(s) d s \quad \text { for } \quad 0<t \leq T,
$$

where $b(t) \geq 0, \beta \geq 0$. Then there is a positive constant $C_{T}$ such that

$$
y(t) \leq b(t)+C_{T} \int_{0}^{t}(t-s)^{-\alpha} b(s) d s \quad \text { for } \quad 0<t \leq T .
$$

LEMMA 2 (Chen et al. [5]). Let $K \in L_{1}(0, T)$. Then for each $\epsilon>0$ there is a positive constant $C_{\epsilon}=C_{\epsilon}\left(\|K\|_{L_{1}(0 . T)}\right)$ such that

$$
\begin{aligned}
& \left|\int_{0}^{T} \int_{0}^{t} K(t-s) f(s) f(t) d s d t\right| \\
& \quad \leq \epsilon \int_{0}^{T} f(t)^{2} d t+C_{\epsilon} \int_{0}^{T}|K(t-s)| \int_{0}^{t} f(s)^{2} d s d t .
\end{aligned}
$$

\section{Stability and convergence}

Both spectral and pseudo-spectral methods are essentially the Ritz- Galerkin methods (combined with some integration formula in the pseudo-spectral case). It is well known that, when Galerkin methods are used, the distance between the exact and the discrete solution (approximation error) is bounded by the distance between the exact solution and its orthogonal projection upon the subspace (projection error), or by the distance between the exact solution and its interpolated polynomial at some suitable points (interpolation error).

In this section, problem (1.1) is discretized only in the space variable. We consider the semidiscrete problem of finding the following semidiscrete approximation $u_{N}(t)$ : $[0, \infty) \mapsto \mathbb{P}_{N}^{0}(\Omega)$ such that

$$
\begin{aligned}
\left(u_{N_{t}}, \chi\right)_{w_{\alpha}} & +A_{w_{\alpha}}\left(u_{N}, \chi\right) \\
& =\int_{0}^{t} K(t-s) B_{w_{\alpha}}\left(u_{N}(s), \chi\right) d s+(f(t), \chi)_{w_{\alpha}}, \quad \forall \chi \in \mathbb{P}_{N}^{0}(\Omega), \\
u_{N}(0) & =P_{N} u_{0},
\end{aligned}
$$


where $A_{w_{\alpha}}(\cdot, \cdot)$ is the bilinear form on $H_{w_{\alpha}, 0}^{1}(\Omega)$ defined by $(2.1)$ and

$$
\begin{aligned}
B_{w_{\alpha}}(u, v) \equiv & B_{w_{\alpha}}(t, s ; u, v) \\
= & \int_{\Omega}\left(\sum_{i, j=1}^{2} b_{i j}(\mathbf{x}, t, s) \frac{\partial u}{\partial x_{i}} \frac{\partial\left(v w_{\alpha}\right)}{\partial x_{j}} \frac{1}{w_{\alpha}}\right. \\
& \left.\quad+\sum_{j=1}^{2} b_{j}(\mathbf{x}, t, s) \frac{\partial u}{\partial x_{j}} v+b_{0}(\mathbf{x}, t, s) u v\right) w_{\alpha}(\mathbf{x}) d \mathbf{x}, \forall u, v \in H_{w_{\alpha}, 0}^{1}(\Omega) .
\end{aligned}
$$

We shall assume that there is a unique generalized solution of (1.1) satisfying the following regularity conditions.

$$
\begin{aligned}
& \mathscr{R} 1: u \in C\left([0, T] ; H_{w_{\alpha}}^{2} \cap H_{w_{\alpha}, 0}^{1}\right), u_{t} \in C\left([0, T] ; L_{w_{\alpha}}^{2}\right) \cap L^{1}\left((0, T] ; H_{w_{\alpha}}^{2} \cap H_{w_{\alpha}, 0}^{1}\right), \\
& \quad u_{t t} \in L^{1}\left([0, T] ; L_{w_{\alpha} .0}^{2}\right) . \\
& \mathscr{R} 2: u \in \mathscr{R} 1 \cap L^{1}\left([0, T] ; H_{w_{\alpha}}^{\sigma} \cap H_{w_{\alpha}, 0}^{1}\right), u_{t} \in L^{1}\left([0, T] ; H_{w_{\alpha}}^{\sigma}\right), \text { for some } \sigma \geq 2 .
\end{aligned}
$$

Chen et al. [5] have examined the theoretical question of existence and uniqueness where the regularity condition $\mathscr{R} 1$ applies with $B$ independent of $t$. Problem (1.1) has limited regularity due to the weakly singular kernel, and there may be some restriction on $\sigma$. Under the regularity assumption $\mathscr{R} 2$, for each $T>0$ and $-1<\alpha \leq 0$, we shall obtain the following error estimate for the spectral approximation of (1.1):

$$
\left\|u_{N}(t)-u(t)\right\|_{w_{\alpha}} \leq C_{T} N^{-\sigma}\left\{\left\|u_{0}\right\|_{\sigma, w_{\alpha}}+\int_{0}^{t}\left\|u_{t}\right\|_{\sigma, w_{\alpha}} d s\right\} \text { for } t \leq T .
$$

First we consider the stability of the semidiscrete scheme (3.1).

THEOREM 1. Let $u_{N}$ be the solution of (3.1). Then there exists a positive constant $C_{T}$ such that

$$
\left\|u_{N}(t)\right\|_{w_{\alpha}} \leq C_{T}\left\{\left\|u_{N}(0)\right\|_{w_{\alpha}}+\int_{0}^{t}\|f(s)\|_{w_{\alpha}} d s\right\}, \quad t \in(0, T] .
$$

ProOF. Taking $\chi$ to be $u_{N}(t)$ from (3.1), then by the coerciveness of $A_{w_{a}}(\cdot, \cdot)$, we have

$$
\begin{aligned}
& \frac{1}{2} \frac{d}{d t}\left\|u_{N}(t)\right\|_{w_{\alpha}}^{2}+c\left\|u_{N}(t)\right\|_{1, w_{\alpha}}^{2} \\
& \quad \leq C\left\{\|f(t)\|_{w_{\alpha}}\left\|u_{N}(t)\right\|_{w_{\alpha}}+\int_{0}^{t} \mid K(t-s)\|\| u_{N}(s)\left\|_{1, w_{\alpha}}\right\| u_{N}(t) \|_{1, w_{\alpha}} d s\right\} .
\end{aligned}
$$

Integrating with respect to $t$ and applying Lemma 2 with suitable $\epsilon$, we obtain

$$
\begin{array}{r}
\left\|u_{N}(t)\right\|_{w_{\alpha}}^{2}\left\|+\int_{0}^{t}\right\| u_{N}(s) \|_{1, w_{\alpha}}^{2} d s \leq C\left\{\left\|u_{N}(0)\right\|_{w_{\alpha}}^{2}+\int_{0}^{t}\|f(s)\|_{w_{\alpha}}\left\|u_{N}(s)\right\|_{w_{\alpha}} d s\right. \\
\left.+\int_{0}^{t}(t-s)^{-\mu} \int_{0}^{s}\left\|u_{N}(\tau)\right\|_{1, w_{\sigma}}^{2} d \tau d s\right\} .
\end{array}
$$


It follows from Lemma 1 that

$\left\|u_{N}(t)\right\|_{w_{\alpha}}^{2}+\int_{0}^{t}\left\|u_{N}(s)\right\|_{1, w_{\alpha}}^{2} d s \leq C\left(\left\|u_{N}(0)\right\|_{w_{\alpha}}+\int_{0}^{t}\|f(s)\|_{w_{\alpha}} d s\right) \sup _{s \leq t}\left\|u_{N}(s)\right\|_{w_{\alpha}}$.

Taking the supremum in both sides with respect to $t$, we have

$$
\left\|u_{N}(t)\right\|_{w_{\alpha}} \leq \sup _{s \leq l}\left\|u_{N}(s)\right\|_{w_{\alpha}} \leq C_{T}\left\{\left\|u_{N}(0)\right\|_{w_{\alpha}}+\int_{0}^{t}\|f(s)\|_{w_{\alpha}} d s\right\} .
$$

We now introduce, following [7], the Ritz-Volterra projection operator $V_{N}^{\alpha}$, defined for an appropriately smooth function $u$ by

$$
\begin{aligned}
& A_{w_{\alpha}}\left(\left(V_{N}^{\alpha} u-u\right)(t), \chi\right) \\
& \quad=\int_{0}^{t} K(t-s) B_{w_{\alpha}}\left(\left(V_{N}^{\alpha} u-u\right)(s), \chi\right) d s, \quad \forall \chi \in \mathbb{P}_{N}^{0}(\Omega), t \geq 0 .
\end{aligned}
$$

We have the following error estimate for the Ritz-Volterra projection.

LEMMA 3. Let $-1<\alpha \leq 0$ and assume that $u \in H_{w_{\alpha}}^{\sigma}(\Omega) \cap H_{w_{\alpha}}^{1}(\Omega)$ and that the second-order partial differential operator $B$ has smooth coefficients. Then we have for the Ritz-Volterra projection operator $V_{N}^{\alpha}$ that

$$
\begin{aligned}
& \left\|\left(V_{N}^{\alpha} u-u\right)(t)\right\|_{w_{\alpha}}+\frac{1}{N}\left\|\left(V_{N}^{\alpha} u-u\right)(t)\right\|_{1, w_{\alpha}} \\
& \quad \leq C N^{-\sigma} \sup _{s \leq t}\|u(s)\|_{\sigma, w_{\alpha}} \leq C N^{-\sigma}\left(\left\|u_{0}\right\|_{\sigma, w_{\alpha}}+\int_{0}^{t}\left\|u_{t}(s)\right\|_{\sigma, w_{\sigma}} d s\right) .
\end{aligned}
$$

PROOF. Let $\rho=V_{N}^{\sigma} u-u$ and $\Pi_{N}$ be the Ritz projection defined by (2.4). From (2.5) we obtain

$$
\left\|\left(\Pi_{N} u-u\right)(t)\right\|_{w_{\alpha}}+\frac{1}{N}\left\|\left(\Pi_{N} u-u\right)(t)\right\|_{1 . w_{\alpha}} \leq C N^{-\sigma}\|u(t)\|_{\sigma, w_{\alpha}} .
$$

Using the definition of the Ritz-Volterra projection and the coerciveness of bilinear form $A_{w_{\alpha}}(\cdot, \cdot)$, we have that with $c>0$

$$
\begin{aligned}
c\left\|\left(V_{N}^{\alpha} u-\Pi_{N} u\right)(t)\right\|_{1, w_{\alpha}}^{2} & \leq A_{w_{\alpha}}\left(\left(V_{N}^{\alpha} u-\Pi_{N} u\right)(t),\left(V_{N}^{\alpha} u-\Pi_{n} u\right)(t)\right) \\
& =A_{w_{\alpha}}\left(\rho(t),\left(V_{N}^{\alpha} u-\Pi_{N} u\right)(t)\right) \\
& =\int_{0}^{t} K(t-s) B_{w_{\alpha}}\left(\rho(s),\left(V_{N}^{\alpha} u-\Pi_{N} u\right)(t) d s\right. \\
& \leq C\left\|\left(V_{N}^{\alpha} u-\Pi_{N} u\right)(t)\right\|_{1, w_{\alpha}} \int_{0}^{t}(t-s)^{-\mu}\|\rho(s)\|_{1, w_{\alpha}} d s
\end{aligned}
$$


and hence

$$
\|\left(\rho(t)\left\|_{1, w_{a}} \leq\right\|\left(\Pi_{N} u-u\right)(t)\left\|_{1, w_{a}}+C \int_{0}^{t}(t-s)^{-\mu}\right\| \rho(s) \|_{1, w_{a}} d s .\right.
$$

Lemma 1 implies

$$
\begin{aligned}
\|\rho(t)\|_{1 . w_{\alpha}} & \leq C_{T} \sup _{s \leq t}\left\|\left(\Pi_{N} u-u\right)(s)\right\|_{1 . w_{\alpha}} \\
& \leq C_{T} N^{1-\sigma} \sup _{s \leq t}\|u(s)\|_{\sigma \cdot w_{\alpha}} \\
& \leq C N^{1-\sigma}\left(\left\|U_{0}\right\|_{\sigma . w_{\alpha}}+\int_{0}^{t}\left\|u_{t}(s)\right\|_{\sigma . w_{\alpha}} d s\right) .
\end{aligned}
$$

We next turn to the $L_{2}$ estimate. By duality arguments, it is well known that for any $\phi \in L_{w_{\alpha}}^{2}(\Omega)$,

$$
\left\|\Phi-\Pi_{N} \phi\right\|_{w_{\alpha}}=\sup _{\|x\|_{w_{\alpha}}=1} \int_{\Omega}\left(\phi-\Pi_{N} \phi\right)(\mathbf{x}) \chi(\mathbf{x}) w_{\alpha}(\mathbf{x}) d \mathbf{x} .
$$

For any given function $g$ with $\|g\|_{w_{\alpha}}=1$, we denote by $\varphi$ the solution of the Dirichlet problem

$$
\begin{aligned}
-\Delta \varphi & =g w_{\alpha} \quad \text { in } \Omega, \\
\varphi & =0 \quad \text { on } \partial \Omega .
\end{aligned}
$$

Since $g w_{\alpha}$ is in $L_{1 / w_{\alpha}}^{2}(\Omega)$, Theorem 3.1 of [1] implies that $\varphi$ belongs to $H_{1 / w_{\alpha}}^{2}(\Omega) \cap$ $H_{1 / w_{\alpha}, 0}^{1}(\Omega)$ and satisfies

$$
\|\varphi\|_{2.1 / w_{\sigma}} \leq C\|g\|_{w_{o}}=C .
$$

Letting $\psi=\varphi / w_{\alpha}$ for each $g \in L_{w_{\alpha}}^{2}(\Omega)$ with $\|g\|_{w_{\alpha}}=1$, we have for $\chi_{N} \in \mathbb{P}_{N}^{0}(\Omega)$,

$$
\begin{aligned}
\int_{\Omega} \rho(\mathbf{x}, t) g w_{\alpha}(\mathbf{x}) d \mathbf{x} & =-\int_{\Omega} \rho(\mathbf{x}, t) \Delta\left(\psi w_{\alpha}\right) d \mathbf{x} \\
& =A_{w_{u}}(\rho(t), \psi) \\
& =A_{w_{\alpha}}\left(\rho(t), \psi-\chi_{N}\right)+A_{w_{s}}\left(\rho(t), \chi_{N}\right),
\end{aligned}
$$

and

$$
\begin{aligned}
A_{w_{a}}\left(\rho(t), \chi_{N}\right)= & \int_{0}^{t} K(t-s) B_{w_{a}}\left(\rho(s), \chi_{N}\right) d s \\
= & \int_{0}^{t} K(t-s)\left\{B_{w_{a}}\left(\rho(s), \chi_{N}-\psi\right)\right. \\
& \left.\quad+\left(\rho(s), B^{*}\left(\psi w_{\alpha}\right) / w_{\alpha}\right)_{w_{a}}\right\} d s .
\end{aligned}
$$


Thus we have

$$
\begin{aligned}
\int_{\Omega} \rho(\mathbf{x}, t) g w_{\alpha}(\mathbf{x}) d \mathbf{x}=C & \sup _{s \leq t}\|\rho(s)\|_{1, w_{\alpha}}\left|\left(\psi-\chi_{N}\right) w_{\alpha}\right|_{1,1 / w_{\alpha}} \\
& +C \int_{0}^{t}(t-s)^{-\mu}\|\rho(s)\|_{w_{u}} d s .
\end{aligned}
$$

Furthermore, due to Lemma 4.7 of [1], for $-1<\alpha \leq 0$ and for any function $\psi w_{\alpha}$ in $H_{1 / w_{\alpha}}^{2}(\Omega) \cap H_{1 / w_{\alpha}, 0}^{1}(\Omega)$, we can choose $\chi_{N} \in \mathbb{P}_{N}^{0}(\Omega)$ so that

$$
\left|\left(\psi-\chi_{N}\right) w_{\alpha}\right|_{1,1 / w_{\alpha}} \leq C N^{-1}\|\varphi\|_{2.1 / w_{\alpha}} .
$$

Hence, applying Lemma 1 to (3.6), we obtain

$$
\begin{aligned}
\|\rho(t)\|_{w_{\alpha}} & \leq C N^{-\sigma} \sup _{s \leq t}\|u(s)\|_{\sigma, w_{\alpha}} \\
& \leq C N^{-\sigma}\left\{\left\|u_{0}\right\|_{\sigma, w_{\alpha}}+\int_{0}^{t}\left\|u_{t}(s)\right\|_{\sigma, w_{\alpha}} d s\right\},
\end{aligned}
$$

which completes the proof.

We also need the following estimate for the time derivative of the error in the Ritz-Volterra projection.

LEMMA 4. Under the assumptions of Lemma 3, we have, for $\rho=V_{N}^{\alpha} u-u$,

$$
\int_{0}^{t}\left(\left\|\rho_{t}\right\|_{w_{\alpha}}+\frac{1}{N}\left\|\rho_{t}\right\|_{1, w_{\alpha}}\right) d s \leq C N^{-\sigma}\left\{\left\|u_{0}\right\|_{\sigma, w_{\alpha}}+\int_{0}^{t}\left\|u_{t}(s)\right\|_{\sigma, w_{o}} d s\right\} .
$$

PROOF. We can rewrite the Ritz-Volterra projection of the form

$$
A_{w_{\alpha}}\left(\rho(t), \chi_{N}\right)=\int_{0}^{t} K(s) B_{w_{\alpha}}\left(t, t-s ; \rho(t-s), \chi_{N}\right) d s, \quad \forall \chi_{N} \in \mathbb{P}_{N}^{0}(\Omega) .
$$

By differentiation with respect to $t$, we obtain that for all $\chi_{N} \in \mathbb{P}_{N}^{0}(\Omega)$,

$$
\begin{aligned}
A_{w_{\alpha}}\left(\rho_{t}(t), \chi_{N}\right)=K(t) B_{w_{\alpha}}\left(t, 0 ; \rho(0), \chi_{N}\right) \\
\quad+\int_{0}^{t} K(s) \frac{d}{d t} B_{w_{\alpha}}\left(t, t-s ; \rho(t-s), \chi_{N}\right) d s .
\end{aligned}
$$

Let $\theta$ be $\left(V_{N}^{\alpha} u-\Pi_{n} u\right)_{t}$. Then with $c>0$

$$
\begin{aligned}
c\|\theta(t)\|_{1, w_{\alpha}}^{2} \leq & A_{w_{\alpha}}\left(\left(V_{N}^{\alpha} u-u\right)_{t}, \theta\right) \\
\leq & C t^{-\mu}\|\rho(0)\|_{1, w_{\alpha}} \cdot\|\theta\|_{1, w_{\alpha}} \\
& \quad+C\|\theta\|_{1, w_{\alpha}} \int_{0}^{t}(t-s)^{-\mu}\left(\|\rho(s)\|_{1, w_{\alpha}}+\left\|\rho_{t}(s)\right\|_{1, w_{\alpha}}\right) d s .
\end{aligned}
$$


Hence, we have

$$
\|\theta(t)\|_{1, w_{\alpha}} \leq C t^{-\mu}\|\rho(0)\|_{w_{\alpha}}+C \int_{0}^{t}(t-s)^{-\mu}\left(\|\rho(s)\|_{1, w_{\alpha}}+\left\|\rho_{t}(s)\right\|_{1, w_{\alpha}}\right) d s
$$

or

$$
\begin{aligned}
\left\|\rho_{t}(t)_{1, w_{\alpha}} \leq C t^{-\mu}\right\| \rho(0)\left\|_{1, w_{\alpha}}+\right\| \Pi_{N} u_{t}-u_{t} \|_{1 . w_{\alpha}} \\
\quad+C \sup _{s \leq t}\|\rho(s)\|_{1 . w_{\alpha}}+C \int_{0}^{t}(t-s)^{-\mu}\left\|\rho_{t}(s)\right\|_{1 . w_{\alpha}} d s \\
\leq C N^{-1-\sigma}\left(t^{-\mu}\left\|u_{0}\right\|_{\sigma . w_{\alpha}}+\left\|u_{t}(t)\right\|_{\sigma . w_{\alpha}}+\sup _{s \leq t}\|u(s)\|_{\sigma, w_{\alpha}}\right) \\
\quad+C \int_{0}^{t}(t-s)^{-\mu}\left\|\rho_{t}(s)\right\|_{1 . w_{\alpha}} d s .
\end{aligned}
$$

Integrating with respect to $t$ and applying Lemma l, we have

$$
\int_{0}^{t}\left\|\rho_{t}(s)\right\|_{1, w_{\alpha}} d s \leq C N^{1-\sigma}\left(\left\|u_{0}\right\|_{\sigma, w_{\alpha}}+\int_{0}^{t}\left\|u_{t}(s)\right\|_{\sigma, w_{\alpha}} d s\right) .
$$

We now turn to the $L_{w_{\alpha}}^{2}$ estimate. Putting $\rho_{t}$ instead of $\rho$ in (3.4), with the notation of Lemma 3, and using (3.7), we obtain

$$
\begin{aligned}
\left(\rho_{t}(t), g\right)_{w_{\alpha}}= & A_{w_{\alpha}}\left(\rho_{t}(t), \psi\right)=A_{w_{\alpha}}\left(\rho_{t}(t), \psi-\chi_{N}\right)+A_{w_{\alpha}}\left(\rho_{t}(t), \chi_{N}\right) \\
= & A_{w_{\alpha}}\left(\rho_{t}(t), \psi-\chi_{N}\right) \\
& +K(t)\left\{B_{w_{\alpha}}\left(t, 0 ; \rho(0), \chi_{N}-\psi\right)+\left(\rho(0), B^{*}(t, 0)\left(\psi w_{\alpha}\right) / w_{\alpha}\right)_{w \alpha}\right\} \\
& +\int_{0}^{t} K(s)\left\{\frac{d}{d t} B_{w_{\alpha}}\left(t, t-s ; \rho(t-s), \chi_{N}-\psi\right)\right. \\
& \left.\quad+\frac{d}{d t}\left(\rho(t-s), B^{*}(t, t-s)\left(\psi w_{\alpha}\right) / w_{\alpha}\right)_{w_{\alpha}}\right\} d s
\end{aligned}
$$

Taking suitable $\chi_{N} \in \mathbb{P}_{N}^{0}(\Omega)$ as in Lemma 3, we see that

$$
\begin{array}{r}
\left\|\rho_{t}\right\|_{w_{\alpha}} \leq C\left\{\frac{1}{N}\left\|\rho_{t}(t)\right\|_{1, w_{\alpha}}+t^{-\mu}\left(N^{-1}\|\rho(0)\|_{1, w_{\alpha}}+\|\rho(0)\|_{w_{\alpha}}\right)\right. \\
+\int_{0}^{t}(t-s)^{-\mu}\left(\frac{1}{N}\left(\left\|\rho_{t}(s)\right\|_{1, w_{\alpha}}+\|\rho(s)\|_{1, w_{\alpha}}\right)\right. \\
\left.\left.+\left\|\rho(s)_{w_{\alpha}}+\right\| \rho_{t}(s) \|_{w_{\alpha}}\right) d s\right\}
\end{array}
$$




$$
\begin{aligned}
\leq & C N^{-\sigma}\left\{\left\|u_{0}\right\|_{\sigma, w_{\alpha}}+t^{-\mu}\left\|u_{0}\right\|_{\sigma, w_{\alpha}}+\sup _{s \leq t}\|u(s)\|_{\sigma, w_{\alpha}}\right\} \\
& +C \frac{1}{N} \int_{0}^{t}(t-s)^{-\mu}\left\|\rho_{t}(s)\right\|_{1, w_{\alpha}} d s+C \int_{0}^{t}(t-s)^{-\mu}\left\|\rho_{t}(s)\right\|_{1 . w_{\alpha}} d s .
\end{aligned}
$$

Integrating with respect to $t$ and applying Lemma 1, we have

$$
\int_{0}^{t}\left\|\rho_{t}(s)\right\|_{w_{\alpha}} d s \leq C N^{-\sigma}\left\{\left\|u_{o}\right\|_{\sigma, w_{\alpha}}+\sup _{s \leq t}\|u(s)\|_{\sigma, w_{\alpha}}\right\}+C \frac{1}{N} \int_{0}^{t}\left\|\rho_{t}(s)\right\|_{1, w_{\alpha}} d s .
$$

Thus we complete the proof from Lemma 3.

THEOREM 2. Let $-1<\alpha \leq 0$ and assume that the solution $u$ of problem (1.1) satisfies the regularity assumption $\mathscr{R} 2$ and that $u_{N}(0)=P_{N} u_{0}$ is chosen so that

$$
\left\|u_{N}(0)-u_{0}\right\|_{w_{\alpha}} \leq C N^{-\sigma}\left\|u_{0}\right\|_{\sigma, w_{\alpha}} .
$$

Then, for each $T>0$, there is a positive constant $C_{T}$ such that the solutions of (1.1) and (3.1) satisfy

$$
\left\|u_{N}(t)-u(t)\right\|_{w_{\alpha}} \leq C_{T} N^{-\sigma}\left(\left\|u_{0}\right\|_{\sigma, w_{\alpha}}+\int_{0}^{t}\left\|u_{t}\right\|_{\sigma, w_{\alpha}} d s\right) \quad \text { for } \quad t \leq T .
$$

PROOF. We write

$$
u_{N}-u=\left(u_{N}-V_{N}^{\alpha} u\right)+\left(V_{N}^{\alpha} u-u\right)=\theta+\rho .
$$

Lemma 3 immediately gives the desired estimate for $\rho$, so it remains to bound $\theta$. From our definitions, we directly have that

$$
\left(\theta_{t}, \chi\right)_{w_{\alpha}}+A_{w_{\alpha}}(\theta, \chi)=\int_{0}^{t} K(t-s) B_{w_{\alpha}}(\theta(s), \chi) d s-\left(\rho_{t}, \chi\right)_{w_{\alpha}}, \quad \forall \chi \in \mathbb{P}_{N}^{0}(\Omega) .
$$

Applying the stability result of Theorem 1 with $u_{N}=\theta$ and $f=-\rho_{t}$, we obtain

$$
\|\theta(t)\|_{w_{\alpha}} \leq C_{T}\left\{\|\theta(0)\|_{w_{\alpha}}+\int_{0}^{t}\left\|\rho_{t}(s)\right\|_{w_{\alpha}} d s\right\}, \quad t \in(0, T] .
$$

Hence, using Lemma 4 and noting $V_{N}^{\alpha} u(0)=\Pi_{N} u_{0}$, that is, $\theta(0)=0$, we have

$$
\|\theta(t)\|_{\omega_{\alpha}} \leq C_{T} N^{-\sigma}\left(\left\|u_{0}\right\|_{\sigma, w_{\alpha}}+\int_{0}^{t}\left\|u_{t}\right\|_{\sigma, w_{\alpha}} d t\right) .
$$

This completes the proof of the desired estimate for $\theta$, and thus the theorem follows. 


\section{Semidiscrete collocation method}

In this section, we discuss the spatial discretization based on the spectral collocation methods with Jacobi weights for $-1<\alpha \leq 0$. Let $\xi_{i}^{\alpha}$ 's be the nodes of the GaussLobatto integration formula of degree $N$ relative to the Jacobi weight $\tilde{w}(\xi)=\left(1-\xi^{2}\right)^{\alpha}$ and $w_{i}^{\alpha}$ the corresponding weight at $\xi_{i}^{\alpha}$. Then, we see that

$$
\int_{-1}^{1} p(\xi) \tilde{w}(\xi) d \xi=\sum_{j=0}^{N} p\left(\xi_{j}^{\alpha}\right) w_{j}^{\alpha} \quad \text { for any } p \in \tilde{\mathbb{P}}_{2 N-1} .
$$

We define the interpolation operator $I_{N}: C^{0}(\bar{\Omega}) \mapsto \mathbb{P}_{N}(\Omega)$ by

$$
I_{N} v\left(x_{i j}^{\alpha}\right)=v\left(x_{i j}^{\alpha}\right), \quad 0 \leq i, j \leq N,
$$

where $x_{i j}^{\alpha}=\left(\xi_{i}^{\alpha}, \xi_{j}^{\alpha}\right)$ for $0 \leq i, j \leq N$. For any real $\mu, \sigma$ such that $0 \leq \mu \leq \sigma$, $\sigma>1$, the interpolation error is estimated as follows (see [4]):

$$
\left\|v-I_{N} v\right\|_{\mu . w_{\alpha}} \leq C N^{2 \mu-\sigma}\|v\|_{\sigma . w_{\alpha}}, \quad \text { for all } v \in H_{w_{\alpha}}^{\sigma}(\Omega) .
$$

Let $B_{N}$ be an approximation to the operator $B$, in which the derivatives are taken via collocation at the points $x_{i j}^{\alpha}$ 's. We have

$$
\begin{aligned}
B_{N} U= & B_{N}(t, s) U \\
=- & \sum_{i, j=1}^{2} \frac{\partial}{\partial x_{j}} I_{N}\left(b_{i j}(\mathbf{x}, t, s) \frac{\partial U}{\partial x_{i}}\right)+\sum_{j=1}^{2} I_{N}\left(b_{j}(\mathbf{x}, t, s) \frac{\partial U}{\partial x_{j}}\right) \\
& \quad+I_{N}\left(b_{0}(\mathbf{x}, t, s) U\right) .
\end{aligned}
$$

Thus our semidiscrete pseudo-spectral approximation of (1.1) is the following collocation problem: We look for a mapping $U \in C^{1}\left(\mathbb{P}_{N}^{0}(\Omega)\right)$ such that, for any $t \in(0, T)$,

$$
\begin{aligned}
U_{t}\left(x_{i j}^{\alpha}\right)-\Delta U\left(x_{i j}^{\alpha}\right)= & \int_{0}^{t} K(t-s) B_{N}(t, s) U\left(x_{i j}^{\alpha}, s\right) d s+f\left(x_{i j}^{\alpha}\right), \\
& 1 \leq i, j \leq N-1, \\
U\left(x_{0 j}^{\alpha}\right)= & U\left(x_{i 0}^{\alpha}\right)=0 \\
U\left(x_{i j}^{\alpha}, 0\right)= & u_{0}\left(x_{i j}^{\alpha}\right), \quad 0 \leq i, j \leq N .
\end{aligned}
$$

We now define a discrete inner product:

$$
(\phi, \psi)_{N}=\sum_{i, j=0}^{N} \phi\left(x_{i j}^{\alpha}\right) \psi\left(x_{i j}^{\alpha}\right) w_{i}^{\alpha} w_{j}^{\alpha}, \quad \forall \phi, \psi \in C^{0}(\bar{\Omega}) .
$$


By (4.1) it follows that

$$
(\phi, \psi)_{N}=(\phi, \psi)_{w_{a}}, \quad \forall \phi, \psi: \phi \cdot \psi \in \mathbb{P}_{2 N-1}(\Omega) .
$$

Moreover,

$$
(\phi, \psi)_{N}=\left(I_{N} \phi, \psi\right)_{N}, \quad \forall \phi, \psi \in C^{0}(\bar{\Omega}) .
$$

Thus, (4.3) can be rewritten as

$$
\left(U_{t}, \phi\right)_{N}+a_{N}(U, \phi)=\int_{0}^{t} K(t-s) B_{N}^{\alpha}(U(s), \phi) d s+(f, \phi)_{N}, \quad \forall \phi \in \mathbb{P}_{N}^{0}(\Omega)
$$

where $a_{N}(\phi, \psi)=\left(\nabla \phi, \nabla\left(\psi w_{\alpha}\right) / w_{\alpha}\right)_{N}$ for any $\phi, \psi \in \mathbb{P}_{N}^{0}(\Omega)$ and

$$
\begin{aligned}
B+N^{\alpha}(\phi, \psi)=\sum_{i, j=1}^{2} & \left(I_{N}\left(b_{i j} \frac{\partial \phi}{\partial x_{i}}\right), \frac{\partial\left(\psi w_{\alpha}\right)}{\partial x_{j}} \frac{1}{w_{\alpha}}\right)_{N}+\sum_{j=1}^{2}\left(I_{N}\left(b_{j} \frac{\partial \phi}{\partial x_{j}}\right), \psi\right)_{N} \\
& +\left(I_{N}\left(b_{0} \phi\right), \psi\right)_{N}, \quad \forall \phi, \psi \in \mathbb{P}_{N}^{0}(\Omega) .
\end{aligned}
$$

The discrete norm

$$
\|\phi\|_{N}=(\phi, \phi)_{N}^{1 / 2}, \quad \forall \phi \in C^{0}(\bar{\Omega})
$$

is equivalent to the $L_{w_{\alpha}}^{2}$-norm, namely

$$
\|\phi\|_{w_{\alpha}} \leq\|\phi\|_{N} \leq 2\|\phi\|_{w_{\alpha}}, \quad \forall \phi \in \mathbb{P}_{N}(\Omega)
$$

(see [3]). It has been proved (see [3]) that the bilinear form $a_{N}(\cdot, \cdot)$ is continuous and coercive over $\mathbb{P}_{N}^{0}(\Omega)$, that is, there exist positive constants $c_{1}, c_{2}$ such that

$$
\left|a_{N}(\phi, \psi)\right| \leq c_{1}\|\nabla \phi\|_{N}\|\nabla \psi\|_{N}, \quad \forall \phi, \psi \in \mathbb{P}_{N}^{0}(\Omega)
$$

and

$$
a_{N}(\phi, \phi) \geq c_{2}\|\nabla \phi\|_{N}^{2}, \quad \forall \phi \in \mathbb{P}_{N}^{0}(\Omega) .
$$

Finally, for any $v \in C^{0}(\bar{\Omega})$, we define $E(v)$ by

$$
(E(v), \phi)=(v, \phi)_{N}-(v, \phi)_{w_{\alpha}}, \quad \forall \phi \in C^{0}(\bar{\Omega}) .
$$

It can be shown (see $[1,3])$ that

$$
|(E(v), \phi)| \leq C\left\{\left\|v-P_{N-1} v\right\|_{w_{\alpha}}+\left\|v-I_{N} v\right\|_{w_{a}}\right\}\|\phi\|_{w_{a}}, \quad \forall \phi \in \mathbb{P}_{N},
$$

from which, for $v \in H_{w_{\alpha}, 0}^{\sigma}(\Omega)$, we have

$$
|(E(v), \phi)| \leq C N^{-\sigma}\|v\|_{\sigma, w_{o}}\|\phi\|_{w_{\alpha}}, \quad \forall \phi \in \mathbb{P}_{N} .
$$

To analyze the convergence properties of the semidiscrete collocation scheme (4.5), we require the following error estimates for the collocation operator $a_{N}(\cdot, \cdot)$ and $B_{N}^{\alpha}(\cdot, \cdot)$. 
LEMMA 5. Let the coefficients of $B$ be sufficiently smooth and $V_{N}^{\alpha}$ be the Ritz-Volterra projection. Then there exists a positive constant $C$ depending on $B$ such that for all $\phi \in \mathbb{P}_{N}^{0}(\Omega)$,

$$
\left|a_{N}\left(V_{N}^{\alpha} u, \phi\right)-A_{w_{\alpha}}\left(V_{N} u, \phi\right)\right| \leq C N^{1-\sigma}\|u\|_{\sigma, w_{\alpha}}\|\phi\|_{N}
$$

and

$$
\left|B_{N}^{\alpha}\left(V_{N}^{\alpha} u, v\right)-B_{w_{\alpha}}\left(V_{N} u, v\right)\right| \leq C N^{1-\sigma}\|u\|_{\sigma, w_{\alpha}}\|v\|_{N}
$$

PROOF. Let $\tilde{u}$ be $V_{N}^{\alpha} u$, and consider for a smooth function $b$,

$$
\left(I_{N}\left(b(\mathbf{x}, t, s) \frac{\partial \tilde{u}}{\partial x_{i}}\right), \phi\right)_{N}-\left(b(\mathbf{x}, t, s) \frac{\partial \tilde{u}}{\partial x_{i}}, \phi\right)_{w_{\alpha}} \quad \text { for } \phi \in \mathbb{P}_{N}(\Omega)
$$

Noting that

$$
I_{N}\left(b \frac{\partial \tilde{u}}{\partial x_{i}}\right)=I_{N}\left(b\left(\frac{\partial \tilde{u}}{\partial x_{i}}-\frac{\partial u}{\partial x_{i}}\right)\right)+I_{N}\left(b \frac{\partial u}{\partial x_{i}}\right)-b \frac{\partial u}{\partial x_{i}}+b \frac{\partial u}{\partial x_{i}}
$$

and using (4.4) and (4.6), we have

$$
\begin{aligned}
& \left|\left(I_{N}\left(b \frac{\partial \tilde{u}}{\partial x_{i}}\right), \phi\right)_{N}-\left(b\left(\frac{\partial \tilde{u}}{\partial x_{i}}\right), \phi\right)_{w_{\alpha}}\right| \\
& \leq\left(\left\|I_{N}\left(b\left(\frac{\partial \tilde{u}}{\partial x_{i}}-\frac{\partial u}{\partial x_{i}}\right)\right)\right\|_{w_{\alpha}}+\left\|b \frac{\partial \tilde{u}}{\partial x_{i}}-\frac{\partial u}{\partial x_{i}}\right\|_{w_{\alpha}}\right)\|\phi\|_{w_{\alpha}} \\
& \quad+\left|\left(E\left(b \frac{\partial u}{\partial x_{i}}\right), \phi\right)\right|
\end{aligned}
$$

From (4.2), (4.8) and Lemma 3, we obtain

$$
\left|\left(I_{N}\left(b \frac{\partial \tilde{u}}{\partial x_{i}}\right), \phi\right)_{N}-\left(b\left(\frac{\partial \tilde{u}}{\partial x_{i}}\right), \phi\right)_{w_{\alpha}}\right| \leq C N^{1-\sigma}\|u\|_{\sigma . w_{\alpha}}\|\phi\|_{w_{\alpha}}
$$

Furthermore (see [1]), $\nabla\left(v w_{\alpha}\right) / w_{\alpha} \in \mathbb{P}_{N}(\Omega)$ for any $v \in \mathbb{P}_{N}^{0}(\Omega)$ and

$$
\left\|\frac{\nabla\left(v w_{\alpha}\right)}{w_{\alpha}}\right\|_{w_{\alpha}} \leq c\|\nabla v\|_{w_{\alpha}} \quad \text { for any } v \in H_{w_{\alpha} .0}^{l}(\Omega)
$$

Thus, taking an appropriate function instead of $b$ in (4.9), we complete the proof. 
THEOREM 3. Let $-1<\alpha \leq 0$. Assume that $f(x, t)$ and the coefficients of $B$ in (1.1) are sufficiently smooth and that the solution $u$ of problem (1.1) satisfies the regularity assumption $\mathscr{R} 2, u(x, \cdot) \in C^{2}(\Omega)$ and $u_{t} \in L^{1}((0, T) ; C(\bar{\Omega}))$. Moreover, assume that the initial data $u_{0}$ is so smooth that

$$
\left\|I_{N} u_{0}-u_{0}\right\|_{w_{\alpha}} \leq C N^{1-\sigma}\left\|u_{0}\right\|_{\sigma, w_{\alpha}} .
$$

Then, for each $T>0$, there is a constant $C_{T}$ such that for the solutions of (1.1) and (4.3),

$$
\|U(t)-u(t)\|_{w_{\alpha}} \leq C_{T} N^{1-\sigma}\left\{\left\|u_{0}\right\|_{\sigma, w_{\alpha}}+\int_{0}^{t}\left\|u_{t}\right\|_{\sigma, w_{\alpha}} d s\right\}+C_{T} \int_{0}^{t}\|E(f(s))\| d s .
$$

PROOF. Let $\tilde{u}=V_{N}^{\alpha} u(t)$ for all $t \geq 0$. The $\tilde{u}$ satisfies the variational equation: for all $\phi \in \mathbb{P}_{N}^{0}(\Omega)$,

$$
\begin{array}{rl}
(\tilde{u}, \phi)_{N}+a_{N}(\tilde{u}, \phi)=\int_{0}^{t} & K(t-s) B_{N}^{\alpha}(\tilde{u}, \phi) d s \\
& +\int_{0}^{t} K(t-s)\left(B_{w_{\alpha}}(\tilde{u}, \phi)-B_{N}^{\alpha}(\tilde{u}, \phi)\right) d s \\
& +\left(\tilde{u}_{t}-u_{t}, \phi\right)_{w_{\alpha}}+\left(E\left(\tilde{u}_{t}\right), \phi\right) \\
& +\left(a_{N}(\tilde{u}, \phi)-A_{w_{\alpha}}(\tilde{u}, \phi)\right)+(f, \phi)_{w_{\alpha}} .
\end{array}
$$

Letting $e(t)=U(t)-\tilde{u}+\tilde{u}-u(t)=\theta(t)+\rho(t)$ for $t \geq 0$ and comparing (4.5) with (4.11), we have

$$
\begin{array}{rl}
\left(\theta_{t}, \phi\right)_{N}+a_{N}(\theta, \phi)=\int_{0}^{t} & K(t-s) B_{N}^{\alpha}(\theta, \phi) d s+(E(f), \phi) \\
& -\left(\tilde{u}_{t}-u t, \phi\right)_{w_{\alpha}}-\left(E\left(\tilde{u}_{t}\right), \phi\right) \\
& +\left\{A_{w_{\alpha}}(\tilde{u}, \phi)-a_{N}(\tilde{u}, \phi)\right\} \\
& +\int_{0}^{t} K(t-s)\left(B_{N}^{\alpha}(\tilde{u}, \phi)-B_{w_{\alpha}}(\tilde{u}, \phi)\right) d s \quad \forall \phi \in \mathbb{P}_{N}^{0}(\Omega) .
\end{array}
$$

Rewrite (4.12) as

$$
\left(\theta_{t}, \phi\right)_{N}+a_{N}(\theta, \phi)=\int_{0}^{t} K(t-s) B_{N}^{\alpha}(\theta, \phi) d s+\sum_{i=1}^{5} I_{i}, \quad \forall \phi \in \mathbb{P}_{N}^{0}(\Omega) .
$$

Using (4.8) and the Cauchy-Schwarz inequalities, we can easily obtain the estimates for $I_{1}, I_{2}$ and $I_{3}$ as

$$
\begin{aligned}
& \left|I_{1}\right| \leq\|E(f)\|\|\phi\|_{N}, \\
& \left|I_{2}\right| \leq C\left\|\rho_{r}\right\|_{\sigma, w_{\alpha}}\|\phi\|_{N}, \\
& \left|I_{3}\right| \leq C N^{1-\sigma}\left\|u_{t}\right\|_{\sigma, w_{\alpha}}\|\phi\|_{N} .
\end{aligned}
$$


From Lemma 7, we have

$$
\left|I_{4}\right|=\left|\left(E(\nabla \tilde{u}), \nabla\left(\phi w_{\alpha}\right) / w_{\alpha}\right)\right| \leq C N^{1-\sigma}\|u\|_{\sigma, w_{u}}\|\phi\|_{1, N}
$$

and

$$
\left|B_{N}^{\alpha}(\tilde{u}, \phi)-B_{w_{\alpha}}(\tilde{u}, \phi)\right| \leq C N^{1-\sigma}\|u\|_{\sigma, w_{g}}\|\phi\|_{1, N} .
$$

Taking $\phi=\theta(t)$ for any $t \geq 0$ in (4.12) and using the estimate (4.14)-(4.18), it follows that

$$
\begin{aligned}
\frac{1}{2} \frac{d}{d t}\|\theta\|_{N}^{2}+c\|\theta\|_{1 . N}^{2} \leq C & \int_{0}^{t}(t-s)^{-\mu}\|\theta(s)\|_{1 . N}^{2} d s+\frac{c}{4}\|\theta(t)\|_{1, N}^{2} \\
& +\sum_{i=1}^{3}\left|I_{i}\right|+C_{T} N^{2(1-a)} \sup _{s \leq 1}\|u\|_{\sigma, w_{\alpha}}^{2}+\frac{c}{4}\|\theta(t)\|_{1 . N}^{2} .
\end{aligned}
$$

Integrating over $t$, we have

$$
\begin{aligned}
\|\theta(t)\|_{w_{\alpha}}^{2} & +c \int_{0}^{t}\|\theta(\tau)\|_{1, N}^{2} d \tau \leq \sup _{s \leq t}\|\theta(s)\|_{w_{\alpha}}\left\{\left\|\theta(0)_{w_{\alpha}}+C \int_{0}^{t}\right\| \rho_{t} \|_{1, w_{\alpha}} d \tau\right. \\
& \left.+C N^{1-\sigma} \int_{0}^{t}\left\|u_{t}\right\|_{\sigma . w_{\alpha}} d \tau+C \int_{0}^{t}\|E(f(s))\| d s\right\} \\
& +C_{T} N^{2(1-\sigma)} \sup _{s \leq t}\|u\|_{\sigma . w_{\alpha}}+C \int_{0}^{t} \int_{0}^{s}(\tau-s)^{-\mu}\|\theta\|_{1 . N}^{2} d s d \tau .
\end{aligned}
$$

Applying Lemma 1, we then have

$$
\begin{gathered}
\|\theta(t)\|_{w_{\alpha}}^{2}+\int_{0}^{t}\|\theta(\tau)\|_{1, N}^{2} d \tau \leq C_{T} N^{2(1-\sigma)} \sup _{s \leq t}\|u\|_{\sigma, w_{\alpha}}^{2}+C_{T} \sup _{s \leq t}\|\theta\|_{N}\left\{\|\theta(0)\|_{N}\right. \\
\left.+\int_{0}^{t}\left\|\rho_{t}\right\|_{1, w_{\alpha}} d \tau+N^{1-\sigma} \int_{0}^{t}\left\|u_{t}\right\|_{\sigma . w_{\alpha}} d \tau+\int_{0}^{t}\|E(f(s))\| d s\right\}
\end{gathered}
$$

Noting that $\|\theta(0)\|_{N}=\left\|\Pi_{N} u_{0}-I_{N} u_{0}\right\|_{w_{\alpha}} \leq C N^{1-\sigma}\left\|u_{0}\right\|_{\sigma-1 . w_{\alpha}}$ and applying Lemma 3 and Lemma 4 , we obtain

$$
\|\theta(t)\|_{N} \leq C_{T} N^{1-\sigma}\left\{\left\|u_{0}\right\|_{\sigma, w_{\alpha}}+\int_{0}^{t}\left\|u_{t}\right\|_{\sigma, w_{\alpha}} d \tau\right\}+C_{T} \int_{0}^{t}\|E(f(s))\| d s .
$$

Now, using the triangle inequality

$$
\|u(t)-U(t)\|_{w_{\alpha}} \leq\|u(t)-\tilde{u}(t)\|_{w_{\alpha}}+\|\theta(t)\|_{w_{\alpha}}, \quad \text { for } t \geq 0
$$

and by Theorem 2, we complete the proof. 


\section{Full discretization schemes}

The semidiscrete approximation (4.3) gives a system of ordinary differential equations in the time variable. Let $h>0$ be a time step, and let $U^{n} \in \mathbb{P}_{N}^{0}(\Omega)$ be the approximation of the exact solution of (1.1) at time $t_{n}=n h$. The time discretization considered will be based on the backward difference quotient

$$
\bar{\partial}_{t} U^{n}=\left(U^{n}-U^{n-1}\right) / h .
$$

For the integral term we use the product integration: we approximate $\phi$ in

$$
J_{n}(\phi)=\int_{0}^{t_{n}} K\left(t_{n}-s\right) \phi(s) d s
$$

by piecewise constant function taking the value $\phi^{j}\left(\equiv \phi\left(t_{j}\right)\right)$ in $\left(t_{j}, t_{j+1}\right)$ and thus we write the quadrature for $J_{n}(\phi)$ as

$$
Q_{n}(\phi)=\sum_{i=0}^{n-1} \int_{t_{j}}^{t_{j+1}} K\left(t_{n}-s\right) \phi\left(t_{j}\right) d s=\sum_{j=0}^{n-1} \kappa_{n-j} \phi\left(t_{j}\right),
$$

where

$$
\kappa_{j}=\int_{t_{j-1}}^{t_{j}} K(s) d s .
$$

For any $\phi \in C^{1}[0, T]$, we can easily show that

$$
\left|\epsilon^{n}(\phi)\right|=\left|Q_{n}(\phi)-J_{n}(\phi)\right| \leq C \sum_{j=0}^{n-1} \tau_{n-j} \int_{t_{j}}^{t_{j+1}}\left|\phi_{t}(s)\right| d s,
$$

where

$$
\tau_{j}=\int_{i_{j-1}}^{t_{j}} s^{-\mu} d s
$$

Our fully discretized scheme is now defined by

$$
\begin{array}{rlrl}
\bar{\partial}_{t} U_{i j}^{n}-\Delta U_{i j}^{n} & =\sum_{k=0}^{n-1} \kappa_{n-j} B_{N}\left(t_{n}, t_{k}\right) U_{i j}^{k}+f_{i j}^{n}, \quad \text { for } 1 \leq i, j \leq N-1, \\
U_{0 j}^{k} & =U_{i 0}^{k}=0 \quad \text { for } 0 \leq i, j \leq N, \quad k \geq 0, \\
U_{i j}^{0} & =u_{0}\left(x_{i j}^{\alpha}\right) \quad \text { for } 0 \leq i, j \leq N, &
\end{array}
$$

where $U_{i j}^{n}=U\left(x_{i j}^{\alpha}, t_{n}\right)$ and $x_{i j}^{\alpha}$ are defined in Section 4. Furthermore, we can rewrite the above as in the variational form

$$
\begin{aligned}
\left(\bar{\partial}_{t} U^{n}, \phi\right)_{N}+a_{N}\left(U^{n}, \phi\right) & =\sum_{j=0}^{n-1} \kappa_{n-j} B_{N}^{\alpha}\left(U^{j}, \phi\right)+\left(f^{n}, \phi\right)_{N}, \quad \forall \phi \in \mathbb{P}_{N}^{0}(\Omega), n \geq 1, \\
U^{0} & =I_{N} u_{0} \quad \text { for } 0 \leq i, j \leq N
\end{aligned}
$$


The following two lemmas are discrete versions of Lemma 1 and Lemma 2. For details, we refer to Chen et al. [5].

LEMMA 6. Let $\left\{w_{n}\right\}$ be a sequence of nonnegative real numbers satisfying

$$
w_{n} \leq \alpha_{n}+\beta \sum_{i=0}^{n-1} \tau_{n-i} w_{i}, \quad n \geq 0,
$$

where $\alpha_{n}, \beta \geq 0$. Then for each $T>0$ there is a positive constant $C_{T}$ such that

$$
w_{n} \leq \alpha_{n}+C_{T} \sum_{i=0}^{n-1} \tau_{n-i} \alpha_{i} \quad \text { for } n k \leq T .
$$

LEMMA 7. Let $\kappa_{n}$ be a sequence of real numbers. Then for each $\epsilon>0$ there is a constant $C_{\epsilon}>0$ such that

$$
\left|\sum_{n=1}^{N} \sum_{j=0}^{n-1} \kappa_{n-j} f_{j} f_{n}\right| \leq \epsilon \sum_{n=1}^{N} f_{n}^{2}+C_{\epsilon} \sum_{n=1}^{N-1}\left|\kappa_{N-n}\right| \sum_{j=0}^{n-1} f_{j}^{2} .
$$

We now give the stability result for the fully discretized scheme (5.2).

THEOREM 4 (Unconditional stability). The scheme (5.2) is stable, namely for any $N$ and any $0<h<1$, there is a positive constant $C_{T}$ such that

$$
\left\|U^{n}\right\|_{N} \leq C_{T}\left(\left\|U^{0}\right\|_{N}+h \sum_{k=1}^{n}\left\|f^{k}\right\|_{N}\right) .
$$

Proof. Take $\phi=U^{n}$ in (5.2). Then we have

$$
\begin{aligned}
& \frac{1}{2}\left(\left\|U^{n}\right\|_{N}^{2}-\left\|U^{n-1}\right\|_{N}^{2}\right)+\operatorname{ch}\left\|U^{n}\right\|_{1 . N}^{2} \\
& \quad \leq C h\left(\sum_{j=0}^{n-1}\left|\kappa_{n-j}\right|\left\|U^{j}\right\|_{1 . N}\left\|U^{n}\right\|_{1 . N}+\left\|f^{n}\right\|_{N}\left\|U^{n}\right\|_{N}\right) .
\end{aligned}
$$

Summing from $n=1$ to $m$ and applying Lemma 7 with suitable $\epsilon$, we have

$$
\begin{aligned}
& \left\|U^{m}\right\|_{N}^{2}+\operatorname{ch} \sum_{n=1}^{m}\left\|U^{n}\right\|_{1, N}^{2} \\
& \quad \leq\left\|U^{0}\right\|_{N}^{2}+C \sum_{n=1}^{m}\left\|f^{n}\right\|_{N}\left\|U^{n}\right\|_{N}+C \sum_{n=1}^{m} \tau_{m-n} h \sum_{j=0}^{n-1}\left\|U^{j}\right\|_{1, N}^{2} .
\end{aligned}
$$


It follows from Lemma 6 that

$$
\left\|U^{m}\right\|_{N}^{2}+h \sum_{n=0}^{m-1}\left\|U^{n}\right\|_{1, N}^{2} \leq C_{T}\left\{\left\|U^{0}\right\|_{N}+h \sum_{n=1}^{m}\left\|f^{n}\right\|_{N}\right\} \max _{n \leq m}\left\|U^{n}\right\|_{N} .
$$

Hence, we have

$$
\left\|U^{m}\right\|_{N} \leq \max _{n \leq m}\left\|U^{n}\right\|_{N} \leq C_{T}\left(\left\|U^{0}\right\|_{N}+h \sum_{n=1}^{m}\left\|f^{n}\right\|_{N}\right) \quad \text { for } m h \leq T .
$$

THEOREM 5 (Convergence). Let $u$ and $\left\{U^{n}\right\}$ be the solutions of (1.1) and (5.2) respectively. Assume that the assumptions of Theorem 3 hold. Further, we assume that $u \in C^{2}\left((0, T) ; C^{1}(\bar{\Omega})\right)$. Then there exists a constant $C_{T}$ independent of $N$ and $h$ such that

$$
\begin{aligned}
\left\|u^{n}-U^{n}\right\|_{w_{\alpha}} \leq & C_{T} N^{1-\sigma}\left(\left\|u_{0}\right\|_{\sigma, w_{\alpha}}+\int_{0}^{t_{n}}\left\|u_{t}\right\|_{\sigma, w_{\alpha}} d s\right)+C_{T} h \sum_{k=1}^{n}\left\|E\left(f^{k}\right)\right\| \\
& +C_{T} h \int_{0}^{t_{n}}\left(\|u\|_{2, w_{\alpha}}+\left\|u_{t}\right\|_{2, w_{\alpha}}+\left\|u_{t t}\right\|_{w_{\alpha}}\right) d s .
\end{aligned}
$$

PROOF. For any $t \geq 0$, we set $\tilde{u}=V_{N}^{\alpha} u, e^{k}=U^{k}-\tilde{u}^{k}+\tilde{u}^{k}-u^{k}=\theta^{k}+\rho^{k}$ and recall that $\tilde{u}$ satisfies (4.11). Subtracting (4.11) from (5.2) at time $t=t_{k}$,

$$
\begin{aligned}
& \left(\bar{\partial}_{t} U^{k}, \phi\right)_{N}-\left(\tilde{u}_{t}^{k}, \phi\right)_{N}+a_{N}\left(\theta^{k}, \phi\right) \\
& =\sum_{j=0}^{k-1} \kappa_{k-j} B_{N}^{\alpha}\left(U^{j}, \phi\right)+\left(E\left(f^{k}\right), \phi\right)-\int_{0}^{t_{k}} K\left(t_{k}-s\right) B_{w_{\alpha}}(\tilde{u}, \phi) d s \\
& \quad-\left(\tilde{u}_{t}^{k}-u_{t}^{k}, \phi\right)_{w_{\alpha}}-\left(E\left(\tilde{u}_{t}^{k}\right), \phi\right)-\left(a_{N}\left(\tilde{u}^{k}, \phi\right)-A_{w_{\alpha}}\left(\tilde{u}^{k}, \phi\right)\right) .
\end{aligned}
$$

It follows that

$$
\begin{aligned}
\left(\bar{\partial}_{t} U^{k}, \phi\right)_{N}+a_{N}\left(\theta^{k}, \phi\right)=\sum_{j=0}^{k-1} & \kappa_{k-j} B_{N}^{\alpha}\left(\theta^{j}, \phi\right)+\left(E\left(f^{k}\right), \phi\right) \\
& +\left\{\left(u_{t}^{k}, \phi\right)-\left(\bar{\partial}_{t} \tilde{u}^{k}, \phi\right)_{N}\right\} \\
& +\left\{a_{N}\left(\tilde{u}^{k}, \phi\right)-A_{w_{\alpha}}\left(\tilde{u}^{k}, \phi\right)\right\} \\
& +\left\{\sum_{j=0}^{k-1} \kappa_{k-j} B_{N}^{\alpha}\left(\tilde{u}^{j}, \phi\right)-\int_{0}^{t_{k}} K\left(t_{k}-s\right) B_{w_{s}}(\tilde{u}, \phi) d s\right\} .
\end{aligned}
$$

We denote $I_{1}, I_{2}$ and $I_{3}$ as follows:

$$
\begin{aligned}
I_{1}^{k}(\phi) & =\left\{\left(u_{t}^{k}, \phi\right)-\left(\bar{\partial}_{t} \tilde{u}^{k}, \phi\right)_{N}\right\} \\
& =\left(u_{t}^{k}-\bar{\partial}_{t} u^{k}, \phi\right)_{w_{\alpha}}-\left(\bar{\partial}_{t} \rho^{k}, \phi\right)_{w_{\alpha}}-\left(E\left(\bar{\partial}_{t} \rho^{k}\right), \phi\right)-\left(E\left(\bar{\partial}_{t} u^{k}\right), \phi\right), \\
I_{2}^{k} & =\left\{a_{N}\left(\tilde{u}^{k}, \phi\right)-A_{w_{\alpha}}\left(\tilde{u}^{k}, \phi\right)\right\}
\end{aligned}
$$


and

$$
\begin{aligned}
I_{3}^{k}(\phi)= & \left\{\sum_{j=0}^{k-1} \kappa_{k-j} B_{N}^{\alpha}\left(\tilde{u}^{j}, \phi\right)-\int_{0}^{t_{k}} K\left(t_{k}-s\right) B_{w_{\alpha}}(\tilde{u}, \phi) d s\right\} \\
= & \epsilon^{k}\left(B_{w_{\alpha}}(\rho, \phi)\right)+\left(B_{w_{\alpha}}(u, \phi)\right) \\
& +\sum_{j=0}^{k-1} \kappa_{k-j}\left\{B_{N}^{\alpha}\left(\tilde{u}^{j}, \phi\right)-B_{w_{\alpha}}\left(\tilde{u}^{j}, \phi\right)\right\} .
\end{aligned}
$$

Taking $\phi=\theta^{k}$ in (5.4), we have

$$
\begin{aligned}
& \frac{1}{2}\left(\left\|\theta^{k}\right\|_{N}^{2}-\left\|\theta^{k-1}\right\|_{N}^{2}\right)+\operatorname{ch} \sum_{k=1}^{n}\left\|\theta^{k}\right\|_{1, N}^{2} \\
& \quad \leq C h \sum_{j=0}^{k-1} \tau_{k-j}\left\|\theta^{j}\right\|_{1, N}\left\|\theta^{k}\right\|_{1, N}+\left\|f^{k}\right\|_{\sigma, w_{\alpha}}\left\|\theta^{k}\right\|_{N}+h \sum_{i=1}^{3}\left|I_{i}^{k}\left(\theta^{k}\right)\right| .
\end{aligned}
$$

Summing (5.5) from $k=1$ to $n$ and applying Lemma 7, we immediately have

$$
\begin{aligned}
& \left\|\theta^{n}\right\|_{N}^{2}+2 c h \sum_{k=1}^{n}\left\|\theta^{k}\right\|_{1, N}^{2} \leq\left\|\theta^{0}\right\|_{N}^{2}+C \sum_{k=1}^{n} \tau_{n-k} \sum_{j=0}^{k-1}\left\|\theta^{j}\right\|_{1, N}^{2}+c \delta h \sum_{k=1}^{n}\|\theta\|_{1, N}^{n} \\
& \quad+2 h \sum_{k=1}^{n}\left\|E\left(f^{k}\right)\right\|_{w_{\alpha}}\left\|\theta^{k}\right\|_{N}+2 h \sum_{k=1}^{n} \sum_{i=1}^{3}\left|I_{i}^{k}\left(\theta^{k}\right)\right| .
\end{aligned}
$$

We now turn to the estimates for $I_{1}, I_{2}$, and $I_{3}$. Since $u \in C^{2}\left((0, T) ; L_{w_{\alpha}}^{2}(\Omega)\right)$, using the Taylor formula with the integral form of the remainder will give

$$
\left|u_{t}^{k}-\bar{\partial}_{t} u^{k}\right| \leq \int_{t_{k-1}}^{t_{k}}\left|u_{t}\right| d s, \quad k \geq 1
$$

and

$$
\mid\left(E\left(\bar{\partial}_{t} \rho^{k}, \phi\right) \mid \leq \frac{1}{h} \int_{t_{k-1}}^{t_{k}}\left\|\rho_{t}(s)\right\|_{w_{\alpha}} d s\|\phi\|_{N} \quad \forall \phi \in \mathbb{P}_{N}^{0}(\Omega) .\right.
$$

Hence, from (4.8), we have immediately

$$
\begin{aligned}
h \sum_{k=1}^{n}\left|I_{1}^{k}\left(\theta^{k}\right)\right| \leq & \sum_{k=1}^{n} \int_{t_{k-1}}^{t_{k}}\left\|u_{t t}(s)\right\|_{w_{\sigma}} d s\left\|\theta^{j}\right\|_{N} \\
& +\sum_{k=1}^{n} \frac{1}{h}\left(\int_{t_{k-1}}^{t_{k}}\left\|\rho_{t}(s)\right\|_{w_{\sigma}} d s+C N^{-\sigma} \int_{t_{k-1}}^{t_{k}}\left\|u_{t}\right\|_{\sigma, w_{\alpha}}\right)\left\|\theta^{k}\right\|_{N} \\
\leq & C h \max _{k \leq n}\left\|\theta^{k}\right\|_{N} \int_{0}^{t_{n}}\left\|u_{t t}(s)\right\|_{w_{\sigma}} d s \\
& +\max _{k \leq n}\left\|\theta^{k}\right\|_{N}\left(\int_{0}^{t_{n}}\left\|\rho_{t}(s)\right\|_{w_{\sigma}}+N^{-\sigma} \int_{0}^{t_{n}}\left\|u_{t}\right\|_{\sigma . w_{\sigma}}\right)
\end{aligned}
$$


Furthermore, using Lemma 7 and the inequality $a b \leq \delta a^{2}+4 / \delta b^{2}$, we have at once the following estimate for $I_{2}$ :

$$
h \sum_{k=1}^{n}\left\|I_{2}^{k}(\phi) \mid \leq C N^{2(1-\sigma)} \max _{k \leq n}\right\| u^{k}\left\|_{\sigma, w_{\alpha}}^{2}+\delta h \sum_{k=1}^{n}\right\| \theta^{k} \|_{1, N}^{2} .
$$

Finally, to estimate $I_{3}$, using the fact that $B_{w_{\alpha}}(u, \phi)=(B u(s), \phi)_{w_{\alpha}}$ and $(5.1)$, we have the following

$$
\begin{aligned}
\left|I_{3}^{k}(\phi)\right| \leq C & \sum_{j=0}^{k-1} \tau_{k-j} \int_{t_{j}}^{t_{j+1}}\left(\|\rho\|_{1, w_{\alpha}}+\left\|\rho_{t}\right\|_{1, w_{\alpha}}\right) d s\|\phi\|_{1, N} \\
& +\sum_{j=0}^{k-1} \tau_{k-j} \int_{t_{j}}^{t_{j+1}}\left(\|u\|_{2, w_{\alpha}}+\left\|u_{t}\right\|_{2, w_{\alpha}}\right) d s\|\phi\|_{N}+C N^{1-\sigma}\left\|u^{k}\right\|_{\sigma, w_{\alpha}}\|\phi\|_{1, N} .
\end{aligned}
$$

Thus we get

$$
\begin{aligned}
h \sum_{k=1}^{n}\left|I_{3}^{k}\left(\theta^{k}\right)\right| \leq & \delta h \sum_{k=1}^{n}\left\|\theta^{k}\right\|_{1, N}^{2}+C h \max _{k \leq n}\left\|\theta^{k}\right\|_{N} \int_{0}^{t_{n}}\left(\|u\|_{2, w_{\alpha}}+\left\|u_{t}\right\|_{2, w_{\alpha}}\right) d s \\
& +C h\left(\int_{0}^{t_{n}}\left(\|\rho\|_{1, w_{\alpha}}+\left\|\rho_{t}\right\|_{1, w_{\alpha}}\right) d s\right)^{2}+C N^{2(1-\sigma)} h \max _{k \leq n}\left\|u^{k}\right\|_{\sigma, w_{\alpha}}^{2} .
\end{aligned}
$$

Thus, (5.6) with suitable $\delta$ gives that

$$
\begin{aligned}
\left\|\theta^{n}\right\|_{N}^{2} & +h \sum_{k=1}^{n}\left\|\theta^{k}\right\|_{1 . N}^{2} \leq C\left\|\theta^{0}\right\|_{N}^{2}+C \sum_{k=1}^{n} \tau_{k-j} \sum_{j=0}^{k-1}\left\|\theta^{j}\right\|_{1 . N} \\
& +C_{T} \max _{k \leq n}\left\|\theta^{k}\right\|_{N}\left\{h \sum_{k=1}^{n}\left\|E\left(f^{k}\right)\right\|_{w_{\alpha}}+N^{-\sigma} \int_{0}^{t_{n}}\left\|u_{t}\right\|_{\sigma, w_{\alpha}} d s\right. \\
& \left.+\int_{0}^{t_{n}}\left\|\rho_{t}(s)\right\|_{w_{\alpha}} d s+h \int_{0}^{t_{n}}\|u\|_{2, w_{\alpha}}+\left\|u_{t}\right\|_{2, w_{\alpha}}+\left\|u_{t t}(s)\right\|_{w_{\alpha}} d s\right\} \\
& +C h\left(\int_{0}^{t_{n}}\left(\|\rho\|_{1 . w_{\alpha}}+\left\|\rho_{t}\right\|_{1, w_{\alpha}}\right) d s\right)^{2}+C N^{2(1-\sigma)} \max _{k \leq n}\left\|u^{k}\right\|_{\sigma, w_{\alpha}}
\end{aligned}
$$


Applying Lemma 6, we obtain that

$$
\begin{aligned}
\left\|\theta^{n}\right\|_{N}^{2} & +h \sum_{k=1}^{n}\left\|\theta^{k}\right\|_{1, N}^{2} \leq C \max _{k \leq n}\left\|\theta^{k}\right\|_{N}\left\{\left\|\theta^{0}\right\|_{N}+h \sum_{k=1}^{n}\left\|E\left(f^{k}\right)\right\|_{w_{\alpha}}\right. \\
& +h \int_{0}^{t_{n}}\left(\|u\|_{2, w_{\alpha}}+\left\|u_{t}\right\|_{2, w_{\alpha}}+\left\|u_{t t}(s)\right\|_{w_{\alpha}}\right) d s \\
& \left.+\int_{0}^{t_{n}}\left\|\rho_{t}(s)\right\|_{w_{\alpha}} d s+N^{-\sigma} \int_{0}^{t_{n}}\left\|u_{t}\right\|_{\sigma, w_{\alpha}} d s\right\} \\
& +C h\left(\int_{0}^{t_{n}}\left(\|\rho\|_{1, w_{\alpha}}+\left\|\rho_{t}\right\|_{1, w_{\alpha}}\right) d s\right)^{2}+C N^{2(1-\sigma)} \max _{k \leq n}\left\|u^{k}\right\|_{\sigma, w_{\sigma}}^{2}
\end{aligned}
$$

It follows from Lemma 3 and Lemma 4 that

$$
\begin{aligned}
\left\|\theta^{n}\right\|_{N} \leq & \sup _{k \leq n}\left\|\theta^{k}\right\|_{N} \\
\leq & C N^{1-\sigma}\left\{\left\|u_{0}\right\|_{\sigma, w_{\alpha}}+\int_{0}^{t_{n}}\left\|u_{\imath}\right\|_{\sigma, w_{\alpha}} d s\right\}+C\left(h \sum_{k=1}^{n}\left\|E\left(f^{k}\right)\right\|_{w_{\alpha}}\right) \\
& \quad+C h \int_{0}^{t_{n}}\left(\|u\|_{2, w_{\alpha}}+\left\|u_{t}\right\|_{2, w_{\alpha}}+\left\|u_{t}\right\|_{w_{\alpha}}\right) d s .
\end{aligned}
$$

This completes the proof.

\section{Acknowledgement}

The authors thank the referee for his comments and suggestions, which led to a much improved version of the paper.

This work was partially supported by KOSEF under grant No 91-08-00-01.

\section{References}

[1] C. Bernardi and Y. Maday, "Properties of some weighted Sobolev spaces and application to spectral approximations", SIAM J. Numer. Anal. 26 (1989) 769-829.

[2] N. Bressan and A. Quarteroni, "Analysis of Chebyshev collocation methods for parabolic equations", SIAM J. Numer. Anal. 23 (1986) 1138-1153.

[3] C. Canuto, M. Y. Hussaini, A. Quarteroni and T. A. Zang, Spectral methods in fluid dynamics (Springer, Berlin, 1987).

[4] C. Canuto and A. Quarteroni, "Approximation results for orthogonal polynomials in Sobolev spaces", Math. Comp. 38 (1982) 67-86. 
[5] C. Chen, V. Thomee and B. Wahlbin, "Finite element approximation of parabolic integrodifferential equation with a weakly singular kernel", Math. Comp. 58 (1992) 587-602.

[6] D. Gottlieb and S. A. Orszag, Numerical analysis of spectral methods: theory and applications (SIAM Philadelphia, PA, 1977).

[7] Y. Lin, V. Thomée and B. Wahlbin, "Ritz-Volterra projections to finite-element spaces and applications to integro-differential and related equations", SIAM J. Numer. Anal. 28 (1991) 1047-1070.

[8] P. Linz, Analytical and numerical methods for Volterra equations (SIAM Philadelphia, PA, 1985).

[9] A. K. Pani, S. K. Chung and R. S. Anderssen, "Convergence of a finite difference scheme for a parabolic integro-differential equation with a weakly singular kernel", CMA Report CMA-MR8-91, Centre for Mathematics and its Application, The Australian National University (1991).

[10] A. K. Pani, S. K. Chung and R. S. Anderssen, "On convergence of finite difference scheme for a parabolic generalized solutions of parabolic and hyperbolic integro-differential equations", CMA Report CMA-MR3-91, Centre for Mathematics and its Application, The Australian National University (1991).

[11] M. Renardy, W. J. Hrusa and J. A. Nohel, Mathematical problems in viscoelasticity, Pitman Monographs and Surveys in Pure and Applied Mathematics No. 35 (Wiley, New York, 1987).

[12] V. Thomée and N. Y. Zhang, "Error estimates for semidiscrete finite element methods for parabolic integro-differential equations", Math. Comp. 53 (1989) 121-139.

[13] E. G. Yanik and G. Fairweather, "Finite element methods for parabolic and hyperbolic partial integro-differential equation", Nonlinear Anal. 12 (1988) 785-809. 